Check for updates

Cite this: Mater. Adv., 2021, 2, 3695

Received 23rd December 2020, Accepted 9th April 2021

DOI: 10.1039/d0ma01010c

rsc.li/materials-advances

\section{The fabrication of a graphene and conductive polymer nanocomposite-coated highly flexible and washable woven thermoelectric nanogenerator $\dagger$}

\author{
Nazakat Ali Khoso, (D) ${ }^{a}$ Guangyu $\mathrm{Xu}^{\mathrm{a}}{ }^{\mathrm{Jiao}} \mathrm{Xie}^{\mathrm{a}}{ }$ Tian Sun $^{\mathrm{b}}$ and Jiajun Wang*c
}

\begin{abstract}
The use of self-powering wearable devices has increased in recent years due to the increasing demands of the internet of things (IOTs). Textile fabrics have shown increasing trends toward wearable electronics due to their outstanding mechanical, electrical, and electronic properties. Herein, we report the development of a highly flexible and washable thermoelectric device with a sheet resistance of 185-45 k $\Omega$. The sheet resistance of the rGO-coated fabric was reduced from 185 to $45 \mathrm{k} \Omega$ on coating with PEDOT:PSS, and was further decreased from $45 \mathrm{k} \Omega$ to $25 \mathrm{k} \Omega$ with 10 padding cycles. The fabric showed a higher wet pickup percentage of $60-80 \%$, which improved the electrical and thermoelectric performance of the resultant textile. The sheet resistance remained stable for up to 20 cycles and then increased ( $25 \Omega-180 \mathrm{k} \Omega$ ). A difference was attained between the human body temperature $\left(T_{h}\right)$ of $36.5^{\circ} \mathrm{C}$ with a temperature gradient $(\Delta T)$ of $16.5{ }^{\circ} \mathrm{C}$ and an ambient temperature of $20.0 \pm 0.5{ }^{\circ} \mathrm{C}$. The performance of the device was significantly improved with an enhanced thermoelectric Seebeck coefficient of 2.5-25.0 $\mu \mathrm{V} \mathrm{K} \mathrm{K}^{-1}$, power factor of $0.25-0.60 \mu \mathrm{W} \mathrm{m} \mathrm{m}^{-1} \mathrm{~K}^{-2}$, and figure of merit $(Z T)$ of $0.02-0.08 \times 10^{-3}$. There was an improvement in the tensile strength of the rGO-coated fabric from 20 to $60 \mathrm{mPa}$, and in the water contact angle (WCA) from $121^{\circ}$ to $152^{\circ}$, with a slightly reduced air permeability of 173 to $165 \mathrm{~cm}^{3} \mathrm{~s}^{2} \mathrm{~s}^{-1}$, without influencing the comfort properties. The study demonstrates that the graphene and polymer nanocomposite-coated textile fabric can be used as a self-powered wearable thermoelectric generator for energy harvesting from low-grade human body heat.
\end{abstract}

\section{Introduction}

Graphene-based wearable textiles have received significant attention from researchers and the scientific community over

\footnotetext{
${ }^{a}$ College of Materials and Textiles, Zhejiang Sci- Tech, University, Zhejiang, Hangzhou, P. R. China

${ }^{b}$ Shanghai Institute of Ceramics, Chinese Academy Sciences (CAS), Shanghai, P. R. China

${ }^{c}$ School of Art and Design at Zhejiang Sci-Tech University, Zhejiang, Hangzhou, P. R. China. E-mail: Wangijhz@163.com

$\dagger$ Electronic supplementary information (ESI) available: Fig. S1. FESEM of rGO and PEDOT: PSS-coated textile fabric at different magnifications, with EDS profile showing elemental analysis (C), (O) \& (S). Fig. S2. Sheet resistance with the number of padding cycles \& washing cycles (a); tensile strength (warp \& weft) (b). Fig. S3. Water contact angle for the fabric coated with GO (a), rGO (b), and PEDOT: PSS-rGO (c). The effect of dyeing cycles on the water contact angle. Fig. S4. TE design model P-N-type legs rGO, rGO/PEDOT: PSS connected in parallel (a), and series (b) arrangements with a closed-circuit. Fig. S5. TE design model (P-N) type legs coated with $\mathrm{rGO}, \mathrm{rGO} / \mathrm{PEDOT}$ : PSS Connected in parallel (a), and series (b) arrangements with a closed-circuit. (a) Obtained electric potential (mV). (b) Seebeck analysis set-up (hot plate). (c) Glowing of the LED. (d) TE device placed on a human wrist. Fig. S1, Supplementary file (S2) and Supplementary file (S3) tables. See DOI: 10.1039/d0ma01010c
}

the last decade after the discovery of graphene as a twodimensional (2D) material with a layer of carbon that is one atomic layer thick. ${ }^{1}$ The demand for graphene-based, highly flexible and breathable, wearable e-textiles is increasing day by day due to their flexibility, stretching and bending ability, and they are considered as highly stable and efficient materials for energy storage, conversion, and harvesting devices. ${ }^{2}$ The conversion of heat released from different industrial appliances, machines and automobiles is considered sustainable energy. ${ }^{3}$ However lowgrade body heat is a new approach for the conversion of energy due to the increasing demand of the internet of things (IOTs) for biomedical and health monitoring applications. ${ }^{3}$ Thermoelectric (TE) devices have been developed, so far, by using various organic and inorganic materials. ${ }^{4}$ These materials include wearable TE devices based on silver $(\mathrm{Ag}),{ }^{5}$ copper $(\mathrm{Cu}),{ }^{6}$ aluminum $(\mathrm{Al}),{ }^{7}$ zinc $(\mathrm{Zn}),{ }^{8}$ gold $(\mathrm{Au}),{ }^{9}$ titanium (TiO2), ${ }^{10}$ selenium $(\mathrm{Se}),{ }^{11}$ selenium telluride (SnTe), ${ }^{12}$ bismuth telluride $(\mathrm{BiTe})^{13}$ and antimony tellurides (SbTe). ${ }^{14}$ The use of such inorganic materials is limited due to their higher rigidity, stiffness, and decomposition when exposed to water and air, and also their poor binding, fixation and washing stability. ${ }^{15,16}$ Additionally, they are toxic to human skin 
and harsh on the environment ${ }^{16-18}$ The fabrication and processing of such rigid materials are complicated and limit their use on a commercial scale. ${ }^{19}$ The use of organic materials such as carbon and its derivatives is therefore highly anticipated for the multifunctional finishing of textiles. ${ }^{20}$ The demand for carbonbased allotropes, including fullerenes, carbon black (CB), carbon nanotubes (CNT), graphite oxide (GrO), graphene oxide (GO), reduced graphene oxide (rGO) and graphene has increased in recent years for use in highly flexible, bendable, breathable and washable e-textiles. ${ }^{21}$ The use of graphene and other twodimensional materials including MXenes, MXenes carbide, and boron nitrides is worth mentioning for potential applications such as biomedical applications, health monitoring, energy harvesting, and storage e-textiles. ${ }^{22}$ The use of intrinsic conductive polymers with graphene is also considered a systematic approach as compared to traditional metal-coated e-textiles. ${ }^{21}$ The graphene-based e-textiles have been widely used for batteries, ${ }^{22}$ supercapacitors, $^{23}$ sensors, $^{24}$ actuators, $^{25}$ solar cells, ${ }^{26}$ triboelectric, ${ }^{27}$ pyroelectric $^{28}{ }^{28}$ piezo-electric, ${ }^{29}$ and thermoelectric generators for electrical energy harvesting. ${ }^{30}$ The fabrication of graphene-based thermoelectric devices is not as complex and challenging as compared to other inorganic materials that need critical care and consideration during processing. ${ }^{31}$ The processes include the chemical and thermal reduction of GO into rGO. ${ }^{32}$ The chemical reduction of GO is generally achieved with strong reducing agents including hydrazine hydrate $(\mathrm{H} . \mathrm{H})$, hydroiodic acid (H.I), and sodium borohydride $\left(\mathrm{NaBH}_{4}\right){ }^{33-35}$ The use of such strong reducing agents is hazardous and limited since they cause damage to human skin and textiles and are carcinogenic. ${ }^{36,37}$ In this context, several studies have been conducted to overcome and reduce these issues by using green reducing agents including, ascorbic acid $\left(\mathrm{C}_{6} \mathrm{H}_{8} \mathrm{O}_{6}\right)$, thiourea $\left(\mathrm{CH}_{4} \mathrm{~N}_{2} \mathrm{~S}\right)$, and sodium hydrosulfite $\left(\mathrm{Na}_{2} \mathrm{~S}_{2} \mathrm{O}_{4}\right){ }^{38-40}$

Thermal reduction of (GO) into rGO is achieved at higher temperatures of $200-400{ }^{\circ} \mathrm{C}$, which is more time-consuming and is not suitable for textile fibers. ${ }^{41}$ Synthetic fibers including polyester, acrylic, lycra, and spandex are susceptible to excessive heat, which results in the deformation of the polymer chains at a higher temperature range above the glass transition $\left(T_{\mathrm{g}}\right)$ 160$200{ }^{\circ} \mathrm{C} .{ }^{42}$ Likewise, the natural fibers, including cotton, jute, flax, and hemp fibers, may also decompose and lose their strength due to the polymer degradation of the cellulose monomer. ${ }^{43}$ Therefore, the use of highly efficient and green reducing agents is needed for the production of rGO-coated textiles. ${ }^{44}$

Different fabrication techniques including; vacuum filtration, ${ }^{45}$ brush painting, ${ }^{46}$ spin coating, ${ }^{47}$ spray coating, ${ }^{48}$ screen printing, ${ }^{49}$ stencil printing, ${ }^{50}$ dip coating, ${ }^{51}$ heat transfer, ${ }^{52}$ vapour deposition, ${ }^{53}$ chemical vapour deposition $(\mathrm{CVD})^{54}$ and wet transfer have been reported in previous studies. ${ }^{55}$ Among these techniques, the pad-dry-cure method is a highly efficient and suitable approach with a production speed of $150 \mathrm{~m} / \mathrm{min}$, which is suitable for the mass production of graphene-coated textiles. ${ }^{56}$ Another challenge during the fabrication of graphene is its hydrophobicity; it cannot be easily dissolved, but can only be dispersed in water and ionic liquids. This hydrophobic nature of graphene also reduces the fixation on textiles and resulting in limited production and low stability against rubbing and washing fastness. ${ }^{57}$ The dispersion of graphene is only possible with the addition of dispersing agents, ${ }^{58}$ ionic and non-ionic liquids, ${ }^{59}$ surfactants including sodium dodecyl sulfonate (SDS), ${ }^{60}$ sodium dodecylbenzene sulfonate (SDBS), ${ }^{61}$ ammonium persulfate (APS), ${ }^{62}$ cetyltrimethylammonium bromide (CTAB), ${ }^{63}$ and hexadecyltrimethylammonium bromide (hCTAB). ${ }^{64}$ The chemical functionalization of graphene is either covalent or non-covalent using ionic and non-ionic liquids. These liquids include ethylene glycol (EG), polyethylene glycol (PEG), glycerol, diethyl glycol (DEG), acetone, phenols, methyl ether ketones (MEK), dimethyl sulfide (DMSO), and dimethyl formamide (DMF). ${ }^{59-62}$ Several binders and thickeners such as polyurethane (PU), polyvinyl alcohol (PVA), polyvinylidene fluoride (PVDF), polystyrene (PS), polystyrene sulfone (PSS), carboxymethylcellulose (CMC), sodium alginate, waterborne polyurethane (WPU) and polyvinylidenes (PVP) have also been used to improve the fixation of graphene on textile fibers. ${ }^{63-71}$

The use of such binders, thickeners and dispersing agents may influence the overall physical properties including, handling, feel, touch, lustre, and end-use properties, for example, electrical, thermal, mechanical and comfort characteristics. Herein, we developed a water-based GO as a dye solution with PEDOT: PSS, which worked as a binder, and PSS worked as a dispersing agent. The application process uses the already commercialized pad-drycure simple dyeing technique approach as a state-of-the-art method towards the fabrication of graphene. We used a commercial-scale technique in the mass-scale fabrication of graphene-coated textile fabrics, with the improved thermoelectric performance of wearable self-powering e-textiles for energy harvesting from low-grade body heat. The GO dyed fabric was chemically and thermally reduced into rGO using a green reducing agent (L-ascorbic acid) at $\left(90{ }^{\circ} \mathrm{C}\right)$ without influencing the comfort properties as a second achievement of the study. This study demonstrates that a pad-dry-cure method is an alternative approach to the development of conductive textiles, which assists towards the better fixation or adhesion and washing fastness of graphene-based fibrous assemblies including woven and nonwoven fabrics without using any binder or thickener.

\section{Experimental}

\subsection{Materials and methods}

Carbon graphite material (Grade 3061) was obtained from Asbury Graphite Mills, USA. (3-4) Polyethylene dioxythiophene, polystyrene sulfonate PEDOT:PSS dispersed in water $\left(\mathrm{H}_{2} \mathrm{O}\right)$ of (1.2) wt\% of a highly conductive grade was purchased from Sigma Aldrich Shanghai. L-Ascorbic acid (Vitamin C) was obtained from Sigma Aldrich, Shanghai, along with ammonia $\left(\mathrm{NH}_{3}\right)$, potassium permanganate $\left(\mathrm{KMnO}_{4}\right)$, sulfuric acid $\left(\mathrm{H}_{2} \mathrm{SO}_{4}\right)$ $99 \%$ purity, hydrogen peroxide $\left(\mathrm{H}_{2} \mathrm{O}_{2}\right) 30 \%$, which were of analytical grade. Cotton fabric $100 \%$ with (65-GSM) and fabric structure of 3/1 plain weave was provided by Hangzhou Textile Dyeing and Finishing Co. Ltd. All the other chemicals were of analytical grade and were used without further modifications. 


\subsection{Synthesis of GO}

Graphene oxide was synthesized using a modified Hummers' method, as reported in the literature and our previous studies. ${ }^{41,73}$ The pure graphite was oxidized in three steps: strong, moderate and high oxidation using sulfuric acid $\left(\mathrm{H}_{2} \mathrm{SO}_{4}\right), \mathrm{KMnO}_{4}$, and hydrogen peroxide $\left(\mathrm{H}_{2} \mathrm{O}_{2}\right)$, respectively. Initially, pristine graphite $(2.5 \mathrm{~g})$ was used for synthesis. Subsequently, $\mathrm{KMnO}_{4}$ solution was developed by dissolving $50 \mathrm{mg}$ of $\mathrm{KMnO}_{4}$ in $10.0 \mathrm{ml}$ DI water and vigorously stirring using a magnetic stirrer at $250 \mathrm{rpm}$ until a homogeneous mixture was formed. During the reaction, the colour of the solution changed from dark to brown. Next, 10-25 ml of hydrogen peroxide $\left(\mathrm{H}_{2} \mathrm{O}_{2}\right)$, a strong oxidizing agent, was added dropwise after raising the temperature to $80-90{ }^{\circ} \mathrm{C}$. The yellow water-based solution of GO was obtained as a final product with a strongly acidic $\mathrm{pH}$ of $2.0-3.0$. The resultant (GO) slurry was separated by centrifuge at $10000 \mathrm{rpm}$ for 20 minutes to neutralize the $\mathrm{pH}$. The as-obtained slurry after neutralization was freeze-dried at $-50{ }^{\circ} \mathrm{C}$ for $48 \mathrm{~h}$ to remove trace water from the GO paste. The freeze-dried GO cake was obtained for further use in preparing the water-based dye solution with different weight percentages of GO.

\subsection{Fabrication}

The fabrication of $100 \%$ woven bleached cotton fabric was accomplished after pre-treatment and cleaning with ethanol. The fabric was weighed and graphene oxide was dissolved in water according to different concentrations by weight $(\mathrm{W} / \mathrm{V})$ of fabric to graphene oxide $\left(2.5,5.0,10.0\right.$, and $\left.20.0 \mathrm{mg} \mathrm{L}^{-1}\right)$. The process was performed in batches using the following steps. In brief, GO powder of different weights (2.5-20.0 $\left.\mathrm{mg} \mathrm{L}^{-1}\right)$ was added to distilled water and ultrasonicated for $30 \mathrm{~min}$ at room temperature to develop GO as a dye solution without using any dispersing agent and binder. The procedure of padding was repeated several times until a sufficient loading of GO was accomplished. The GO-coated fabric was dried at $60{ }^{\circ} \mathrm{C}$ after each padding cycle and chemically reduced to rGO using a green reducing agent (L-ascorbic acid) at $90{ }^{\circ} \mathrm{C}$ via microwaveassisted chemical and thermal reduction; ${ }^{42}$ see $\mathrm{ESI} \dagger$ (ESI-S5 $\dagger$ Fig. S1).

2.3.1 Padding. The aqueous water-based GO as the dye solution was loaded in the fabrication bath with a fabric to liquor ratio of $1: 40$, by varying the weight percent of $\operatorname{GO}(2.5,5.0$, 10.0 and $\left.20.0 \mathrm{mg} \mathrm{L}^{-1}\right)$. The fabric was dipped and simultaneously squeezed during each padding cycle to remove the excessive amount of GO solution from the fabric surface with a pneumatic pressure of $15 \mathrm{~kg} / \mathrm{cm}^{2}$ and processing speed of $15 \mathrm{~m} / \mathrm{min}$ as reported in the literature. ${ }^{43}$

2.3.2 Drying. The as coated fabric with GO was thermally dried in a microwave oven at $60{ }^{\circ} \mathrm{C}$ for $15-30 \mathrm{~min}$ after each dip of the fabric.

2.3.3 Reduction. The same procedure was repeated for 10 dips and followed by the reduction of GO coated fabric into rGO using green, reducing agent vitamin C (10\%) L-ascorbic acid at $90{ }^{\circ} \mathrm{C}$ as reported in our previous studies. ${ }^{44}$
2.3.4 Curing or fixation. The rGO-coated fabric was cured at $140{ }^{\circ} \mathrm{C}$ for $2.5 \mathrm{~min}$ using a microwave oven.

2.3.5 Washing and cleaning. The rGO-coated fabric was washed with plenty of water to remove the traces of the reducing agent and unfixed graphene nanoflakes from the fiber surface.

2.3.6 Coating of the conductive polymer. The conductive polymer was applied as rGO coated textile electrodes using a layer-by-layer approach. The same approach was used for the fabrication of the conductive polymer (PEDOT: PSS). After 2-5 dip coatings of the conductive polymer fabric, it was dried at (60$70{ }^{\circ} \mathrm{C}$ ) for $10-15$ minutes.

\subsection{Design of the thermoelectric (TE) device}

The thermoelectric device was fabricated by cutting the fabric strips with each leg size of $(0.5 \times 5.0 \mathrm{~cm})$ and connecting them with each other using adhesive copper tape. The electrical and thermal conductivity and Seebeck coefficient of the developed TE device were measured using a digital Keithley device. The real-time monitoring and performance of the wearable TE device were demonstrated by placing the device on a human wrist and palm.

Fig. 1(d) and (e) show the as-developed TE device for realtime performance measurement in terms of sheet resistance in the folded and normal positions, respectively. The as-coated rGO and PEDOT:PSS-rGO legs were connected with adhesive copper tape from top to bottom as presented in blue and black as shown in Fig. 1(h); the PEDOT: PSS and rGO coated fabric strips are shown. The as-developed TE device demonstrated thermoelectric performance with open-circuit output volts $(\mathrm{mV})$ and AC current when directly placed next to human skin (Fig. 1f and $\mathrm{g}$ ). The TE device model is given in Fig. $1 \mathrm{~h}$. The fabricated samples of fabric with GO, and reduced to rGO (shown in Fig. 1a and b, respectively, were cut into identical strips of size $0.5 \times 5.0 \mathrm{~cm}$, as shown in Fig. 1c. The electrical performance of the TE device was measured before being connected as TE legs using adhesive copper tape and placed on the human wrist as depicted in Fig. 1(d)-(f). The proposed TE model design is

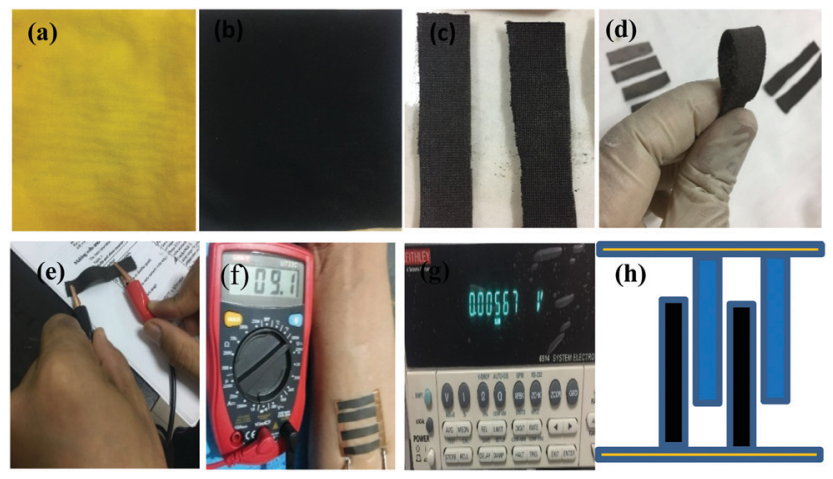

Fig. 1 (a and b) GO and rGO-coated cotton fabric; (c) textile strips; (d) bending of the textile fabric strips; (e) measurement of the sheet resistance of the fabric strip with bending. (f) Real-time performance measurement for the TE device placed on the human wrist. (g) Electric potential as an output voltage (mV). (h) TE design model. 
presented in Fig. $1 \mathrm{~g}$ and $\mathrm{h}$ connected to a digital device. The fabrication of graphene oxide on the textile substrate using the pad-dry-cure method is given in the ESI $\dagger$ (ESI-S3) fabrication methods.

\section{Results and discussion}

\subsection{Measurements and characterization}

The elemental analysis of the graphene oxide, reduced graphene oxide, and PEDOT: PSS-rGO was conducted using FTIR, Raman, XRD and XPS spectroscopy. K-alpha mode on XPS (Thermo Fisher USA), X-ray diffraction (XRD), and (XPS) were used to describe the rGO, GO, and PEDOT: PSS-rGO films and nanocomposites; see the ESI $\dagger$ (ESI-S2) characterization section. The scanning electron microscopy (SEM) analysis of the sample was performed by using a Zeiss ultra-scanning field emission scanning electron microscope (FESEM) for the surface characterization of the treated and untreated fabrics. The electrical performances of the rGO and PEDOT PSS-decorated cotton fabric were measured using a four-point probe (SZT-2B) system (Suzhou Genisis Electronics Co. Ltd China); ${ }^{45}$ see the ESI $\dagger$ (ESI-S3) performance measurement section. The thermoelectric properties such as the Seebeck coefficient and power factor of the TE device were measured according to ASTM standard E-977- $05^{46}$ based on a hot plate and 2-probe thermocouples connected to a digital voltmeter (Keysight). The temperature gradient was measured with a fixed temperature of $20{ }^{\circ} \mathrm{C}$ and a variable temperature was detected using a needle transducer thermocouple by placing the specimen on the hot plate for the temperature difference; see the ESI $\dagger$ (ESI-S3) performance measurement section. The thermal conductivity of the textile fabric samples was measured using an infra-red IR-non-contact device, according to ASTM standard D-7984-16. ${ }^{47}$ All the tests were performed under standard testing conditions for textiles according to ASTM standards-D-1776/D-1776-M-20 ${ }^{48}$ at ambient temperature $\left(25 \pm 5{ }^{\circ} \mathrm{C}\right)$ and relative humidity of $65 \pm 5 \%$.

3.1.1 FESEM and EDS. The morphological studies of the resultant rGO-coated textile substrate are illustrated in Fig. 2(a)-(c), showing that the coating of rGO was accomplished with a significant amount of carbon content, which was determined from EDS analysis with FESEM. The conductive polymer was successfully coated on the rGO-coated fabric as layer by layer (LbL) as reported in previous studies. ${ }^{43}$ From the FESEM results, it was observed that the entire surface of the fiber was coated and covered with polymer forming a smooth surface, showing strong binding and adhesion of the conductive polymer with rGO and a more conductive path between the fiberfiber welding in the yarn assembly as shown in the colour mapping of SEM images Fig. 2(d)-(f). The fiber surface in the yarn was entirely covered with conductive polymer and rGO nanofilms due to the strong adhesion and bonding; this effect may be attributed to the negatively charged polystyrene (PSS) groups in the conjugated polymer PEDOT:PSS. ${ }^{44}$

3.1.2 XRD analysis. The elemental analysis of the as coated rGO fabric was performed using XRD to identify the crystalline
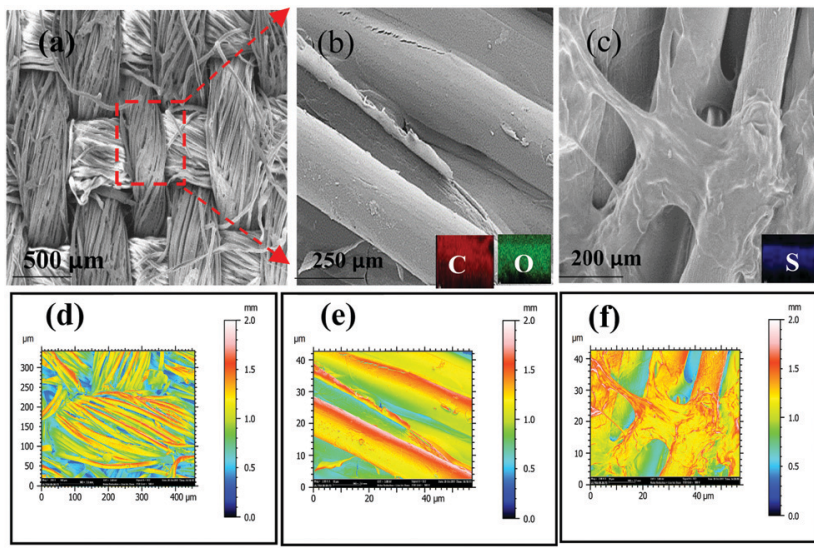

Fig. 2 FESEM images of rGO-PEDOT:PSS film-coated fabric at different magnifications: (a) $500 \mu \mathrm{m}$, (b) $250 \mu \mathrm{m}$ for the rGO-coated fabric, and (c) $200 \mu \mathrm{m}$ coated with rGO-PEDOT:PSS. (d-f) Colour mapping of SEM images IR-rendering using the Mountains map software.

regions of GO, rGO and PEDOT:PSS-rGO, respectively. The X-ray diffraction results showed that firm peaks for GO $2 \theta$ were located at $9.5^{\circ}$. The $2 \theta$ diffraction angle shifted from $9.5^{\circ}$ to $16.5^{\circ}$, which indicated that the graphene oxide was successfully converted into reduced graphene oxide, as shown in Fig. 2(b). The sharp XRD peaks of pristine graphite observed at $28.4^{\circ}$ were reduced when graphitic oxide was converted into GO, and were further reduced to $19.5^{\circ}$ (rGO) due to the restacking of graphene sheets similar to graphite. These broader and comprehensive peaks became widened, which may be attributed to an increase in the particle size of rGO films, as compared to pure graphite and graphitic oxide (GO) as shown in Fig. 2(a) and (b). The second small sharp peak was observed at $19.8^{\circ}$ and may be attributed to the formation of PEDOT:PSS crystals. The very small peak located at $25.6^{\circ}$ represented polymer (PEDOT:PSS) as shown in Fig. 2(d); see the ESI $\dagger$ (ESI-S1 Fig. S1). ${ }^{49}$ The XRD diffraction analysis showed that the oxidized GO had intensity peaks $\left(2 \theta=9.5^{\circ}\right)$ with crystal planes of $(001)$. The peak was shifted to $2 \theta$ of $9.5^{\circ}$ with $d$-spacing of $0.835 \mathrm{~nm}$ due to the higher oxidation level of GO as compared to pure graphite as shown in Fig. 3(a) and (b), respectively. The diffraction peaks shifted towards $2 \theta=14.5^{\circ}$ crystal planes of (002) as chemical and thermal reduction was attained. The resultant peaks became shorter and shifted towards $(2 \theta=19.4)$ with the $d$-spacing of (002), showing reduced (rGO) intensity as compared to the pristine graphite $\left(2 \theta=28.5^{\circ}\right)$. As GO was reduced to rGO, the peak became wider and was reduced, with a $d$-spacing of $d=0.36$ to $d=0.353$ with the (002) crystal plane as compared to pristine graphite with a $d$-spacing of $d=0.339 \mathrm{~nm}$. The peaks that appeared with GO shifted from $2 \theta=16.5^{\circ} \& 19.5^{\circ}$ and this is attributed to polymer crystal formation. Very small and wide peaks were also observed at $2 \theta=26.5^{\circ}$ and $2 \theta=34.5^{\circ}$, which are attributed to the crystal growth of rGO films and PEDOT:PSS after thermal annealing and chemical reduction. ${ }^{50}$

3.1.3 FTIR analysis. FTIR studies were conducted on GO, rGO, and PEDOT:PSS, as well as nanocomposites of the PEDOT:PSS-rGOcoated textile fabric with several functional groups present in rGO 

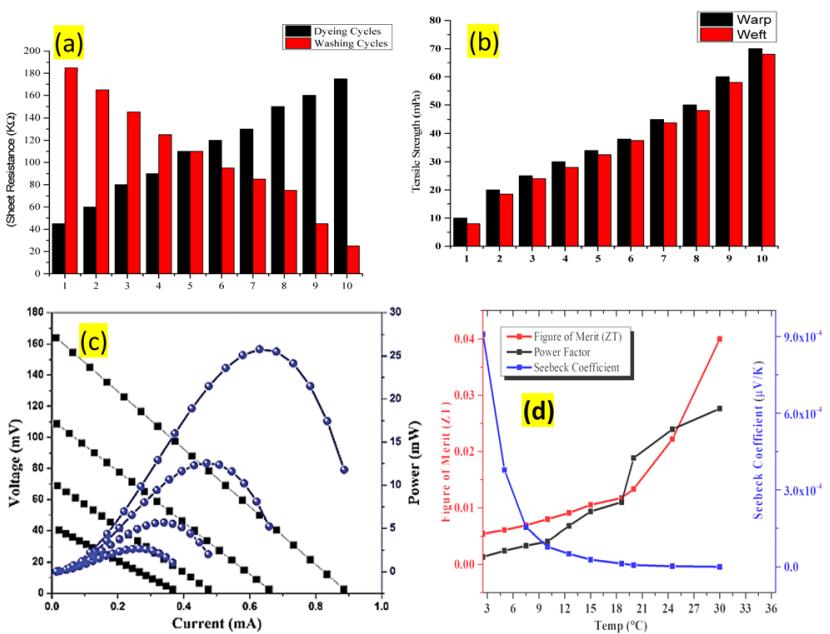

Fig. 3 Sheet resistance with the number of dyeing cycles (a) and washing cycles. (b) Tensile strength (warp and weft). (c) Current vs. voltage relationship of the thermoelectric device with the number of legs with a variable temperature gradient of $2.5-16.5{ }^{\circ} \mathrm{C}$. (d) Overall performance of the TE device including the figure of merit, Seebeck coefficient, and power factor with a temperature gradient of $2.6-36.5^{\circ} \mathrm{C}$.

and PEDOT: PSS. The broader spectral peaks positioned at $3450 \mathrm{~cm}^{-1}$ were reduced for rGO as compared to natural cotton and the GO-coated textile substrate. The development of new functional groups located at $1350 \mathrm{~cm}^{-1}, 1665 \mathrm{~cm}^{-1}$ and $1730 \mathrm{~cm}^{-1}$ showed the development of different functional groups with five chemical shifts as shown in Fig. 2; see ESI $\dagger$ (ESI-S1 Fig. S2). The de-convoluted reactive groups were assigned to the vibrations of the absorbing water molecules hydroxyl $\left(\mathrm{OH}^{-}\right)$groups positioned at $\left(3400 \mathrm{~cm}^{-1}\right)$, carbonyl $\mathrm{C}=\mathrm{O}\left(1740 \mathrm{~cm}^{-1}\right)$, carbonyl $\left(\mathrm{OH}^{-}\right)$deformation peak $\left(1420 \mathrm{~cm}^{-1}\right)$, carboxyl C-OH $\left(1220 \mathrm{~cm}^{-1}\right)$, epoxy groups $\mathrm{C}-\mathrm{O}\left(1050 \mathrm{~cm}^{-1}\right)$ and $1620 \mathrm{~cm}^{-1} \cdot 51$

3.1.4 Raman analysis. The reman spectra showed two broad and identical D and G bands demonstrating the highly oxidized two-dimensional hexagonal structures of carbon atoms for GO. The visual effects of the annealing temperature on graphene oxide (GO) show first order and second order (D and G) vibrational bands. These peaks at $1345 \mathrm{~cm}^{-1}$ and $1580 \mathrm{~cm}^{-1}$ are attributed to the reduction of oxygen-containing functional groups as compared to graphite and GO with $1560 \mathrm{~cm}^{-1}$ as shown in Fig. S4(a) and (b); see ESI $\dagger$ (ESI-S1 Fig. S3). ${ }^{52}$ The ID/IG ratios of the D and $\mathrm{G}$ bands were improved from $0.80,0.85$, and 0.96 for GO, rGO and PEDOT: PSS-rGO respectively. The changes are attributed to the decrease of the oxygen $(\mathrm{C}-\mathrm{O})$, carboxyl $(\mathrm{C}=\mathrm{C})$, carbonyl $(\mathrm{C}=\mathrm{C})$ and epoxide $(\mathrm{C}-\mathrm{O}=\mathrm{C})$ functional groups after the reduction of GO into rGO, as shown in Fig. S4(c) and (d); see the ESI $\dagger$ (ESI-S1 Fig. S3). The results show that the oxidized state of GO was changed from the first-order scattering of $\mathrm{sp} 3$ to $\mathrm{sp} 2$ hybridization and due to the restacking of the carbon atoms of rGO during chemical ad thermal annealing as reported in literature. ${ }^{53}$ Dangling bands occurred between the D and G-bands of rGO, which may be attributed to the presence of sulfone (SP2) groups present in PEDOT: PSS films as shown in Fig. S4d, ESI†.
3.1.5 XPS analysis. The XPS elemental analysis (C1s) and (O1s) spectra and survey peaks are presented in the ESI $\dagger$ (ESI-S2), characterization section, Fig. S3. The results show the presence of the carbon and oxygen groups for different bonds such as $(\mathrm{C}-\mathrm{C})$ and $(\mathrm{C}=\mathrm{C})$. The broader peaks show the $(\mathrm{C}=\mathrm{C})$ bond structure located at 284.7, 284.2, and the $(\mathrm{C}-\mathrm{C})$ peaks located at 284.2 and 285.4. Another functional group $(\mathrm{C}=\mathrm{O})$ was observed at 287.2 and 286.4. Shallow peaks at 289.0 confirmed the $(\mathrm{O}=\mathrm{C}-\mathrm{O})$ functional groups after the reduction of GO into rGO. ${ }^{54}$ These chemical groups indicated the substantial reduction of graphene oxide into rGO. ${ }^{49}$ The survey peaks showed the presence of the carbon (C) and oxygen (O) groups; see ESI $\dagger$ (ESI-S1 Fig. S4)

\section{Measurements}

The textile-based TE device was fabricated after cutting the coated rGO and PEDOT: PSS strips with a specimen size of $(0.5 \times 2.5 \mathrm{~cm})$. The arrangement of TE legs was connected by adhesive copper tape in parallel and series arrangements. The open-circuit voltage was measured using parallel and series arrangements as reported in previous studies. ${ }^{55}$ The legs were connected with conductive adhesive tape to develop heterojunctions. The resultant TE device was placed on a human wrist to measure the thermoelectric performance without any masking tape as shown in Fig. 1(a)-(d), respectively.

\subsection{Electrical and thermoelectric (TE) performance}

The overall electrical conductivity was enhanced by increasing the percentage of rGO as the filler in the conductive polymer, whereas the thermal conductivity was reduced with the number of dyeing or padding passes. The study shows that electrical and thermal conductivity increased and decreased, respectively, with the number of coating layers on the textile substrate. The overall TE performance of the resultant textile electrode was significantly improved for the textile substrate coated with PEDO:PSS-rGO as compared to pristine rGO. The results are in strong agreement with recent works with rGO and PEDOT:PSS using the layer-by-layer approach. ${ }^{56}$ The improvement in the electrical conductivity may be attributed to increasing the number of graphene layers on the textile substrate, which may occur due to the interlayers connected fibers in the yarn structure and fabric. The result showed that thermal conductivity decreased as the number of coating layers decreased, which may occur due to the synergic effect between the graphene and conductive polymer layers. ${ }^{57}$

The electrical conductivity was measured according to ASTM standard D-257 ${ }^{58}$ for polymer-coated conducive textiles. The electrical conductivity was studied in terms of the sheet resistance (Rs) of the textile substrate coated with rGO, PEDOT:PSS, and rGO-PES at a different weight percent of rGO. The average test results with a low sheet resistance of each sample were measured six times and recorded for statistical analysis. The heat transport phenomenon may be attributed to the vibrational movement of free heat carriers (phonons) and charge carrier electrons 
between the graphene layers. The vibrational movements of charge carrier electrons and heat carrier phonons result in the improvement of the thermoelectric effect, with improving the Seebeck coefficient, and power factor figure of merit (zT) value as demonstrated in the ESI, $\dagger$ EIS-S1 Table S2. Fig. 3(c) and (d) demonstrate that the thermoelectric performance of the resultant textile electrode was enhanced the electric potential $(\Delta V)$ due to charge carrier (p-type) graphene and heat carrier phonons in the rGO as p-doped, and PEDOT: PSS as n-type materials.

The change in temperature $\left(T_{2}\right)$ and $\left(T_{1}\right)$ was with respect to a hot side next to the human body, and a cold side exposed to the external environment. ${ }^{58}$ The temperature was raised to $36.5{ }^{\circ} \mathrm{C}$ and ambient working conditions of $20{ }^{\circ} \mathrm{C}$ resulted in the electric potential of 2.5-9.5 $\mathrm{mV}$ for 2-4 thermoelectric legs with a temperature gradient $\Delta T 309 \mathrm{~K}$. The results show that as the number of TE legs were increased to 2-4 legs and connected in parallel and series arrangements, the open circuit output increased from 9.5 to $12.5 \mathrm{mV}$. The coated textile fabric showed the improved thermoelectric potential of 12.5-19.5 mV, which was further raised to $19.5-45.0 \mathrm{mV}$ for parallel and to 45.5$120.0 \mathrm{mV}$ when the TE device was directly attached to the human wrist without any surface coating, which is several times higher as compared to reported textile-based TE devices. ${ }^{59}$ The resultant TE device showed an increased Seebeck coefficient (0.5-9.0 $\left.\times 10^{-4} \mu \mathrm{V} \mathrm{K}^{-1}\right)$, power factor $\left(0.5-0.25 \mathrm{~mW} \mathrm{~m}^{-1} \mathrm{~K}^{-2}\right)$, and dimensionless figure of merit $\left(0.02-0.04 \times 10^{-3}\right)$, as shown in the ESI $\dagger$ (ESI-S1) Table 1. The results show that the dimensionless figure of merit was enhanced as the temperature increased from $20{ }^{\circ} \mathrm{C}$ for the cold side $\left(T_{\mathrm{c}}\right)$ and $36.5{ }^{\circ} \mathrm{C}$ for the hot side $\left(T_{\mathrm{h}}\right)$. The fabric was successfully decorated with p-type and n-type hetrojunctions as a TE nanogenerator for the conversion of human body heat into an electrical response. The resultant textile-based TE device showed improved thermoelectric performance and was capable of directly converting the heat energy into electrical energy in both hot and cold climatic conditions. ${ }^{59}$

\subsection{Textile properties}

4.2.1 Washing stability. The washing tests were performed according to the ASTM standard of ASTM D435-42(1955) ${ }^{60}$ by using a common detergent and a home laundry machine. The performance of the samples was analysed using repeated washing of 20 cycles. The colour faded from black to light brown. The electrical performance of the fabric was assessed before washing and after each wash in terms of electrical sheet resistance. The fabric showed slightly reduced sheet resistance as mentioned in the ESI $\dagger$ (ESI-S1 Table S1 and Fig. S1 ESI-S2). On the other hand, the graphene layers may be removed from the fiber assemblies during washing cycles, resulting in increased sheet resistance. The sheet resistance decreased from $185 \mathrm{k} \Omega$ to $125 \mathrm{k} \Omega$, which may be attributed to the number of graphene layers with increasing the padding passes and coating of PEDOT: PSS nanocomposites. The sheet resistance decreased on increasing the number of washing cycles. The results are presented in the ESI, $\dagger$ Table S2. The air permeability, tensile strength and fabric thickness also decreased with increasing the number of dyeing and washing cycles; ${ }^{61}$ see the ESI $\dagger$ (ESI-S1 Tables S1 and S2). The results also demonstrate that the thermoelectric performance of the resultant textile electrodes was improved with increasing the content of rGO as filler in the conductive polymer PEDOT:PSS, resulting in improved thermoelectric effects; i.e. Seebeck effect, power factor, and figure of merit.

4.2.2 Air permeability. The air permeability of the coated samples of GO, rGO, and PEDOT: PSS-rGO were analysed according to ASTM standards D-737-96. ${ }^{62}$ Each sample was studied before dyeing and the washing cycles to measure the breathability of the as-coated textile substrates. The air permeability of the uncoated samples decreased with the number of dyeing cycles as presented in the ESI $\dagger$ (ESI-S1, see Table 2). The air permeability further decreased as the number of dyeing cycles increased, which may be attributed to the number of layers of rGO and PEDOT:PSS. PEDOT significantly influenced the air permeability as compared to pristine GO and rGO coatings, without influencing the breathability of the comfort properties without any compromise in moisture transport and breathability.

4.2.3 Water contact angle (WCA). The hydrophobic and hydrophilic behaviours of the textile substrate samples before and after coating with the GO, rGO, and PEDOT: PSS-rGO were analysed with a digital contact angle analyser using deionized DI water to measure the contact angle of the as-coated textile fabric according to the ASTM standard D-5725. ${ }^{63}$ The measurement determined the wettability of the textile fabric surface before and after coating. The study demonstrated that the water contact angle (WCA) increased as the content of rGO was increased with the number of dyeing cycles. The maximum contact angles of $121.3^{\circ}$ and $141.5^{\circ}$ were obtained with 10 padding passes; see ESI $\dagger$ (ESI-S1, Table S2). The rGO-coated textile fabric showed higher water resistance and lowered the wettability as compared to natural cotton fabric and the GO-coated fabric sample. It was observed that the samples coated with rGO and the PEDOT:PSS nanocomposites showed

Table 1 The effect of the percentage of rGO content on fabric thickness after a number of padding passes and its effect on the electrical and thermoelectric properties

\begin{tabular}{lllllr}
\hline Sample no. & $\begin{array}{l}\text { Comp } \\
(\mathrm{rGO} \%)\end{array}$ & $\begin{array}{l}\text { Sheet } \\
\text { resistance }(\mathrm{k} \Omega)\end{array}$ & $\begin{array}{l}\text { Seebeck coefficient } \\
\left(\mu \mathrm{V} \mathrm{K} \mathrm{K}^{-1}\right) \times 10^{-4}\end{array}$ & $\begin{array}{l}\text { Power factor } \\
\left(\mu \mathrm{W} \mathrm{m}^{-1} \mathrm{~K}^{-2}\right)\end{array}$ \\
\hline rGO/PES-1 & 2.5 & $185 \pm 5$ & 2.5 & 0.5 & $\begin{array}{l}\text { Figure of merit } \\
Z T \text { value } 10^{-3}\end{array}$ \\
rGO/PES-2 & 5.0 & $165 \pm 5$ & 12.5 & 0.15 & 0.01 \\
rGO/PES-3 & 10.0 & $145 \pm 5$ & 18.0 & 0.20 & 0.02 \\
rGO/PES-4 & 20.0 & $125 \pm 5$ & 25.5 & 0.25 & 0.03
\end{tabular}


Table 2 The effects of different weight percent of rGO on the sheet resistance before and after washing cycles, tensile strength and air permeability of the as-coated fabric with nanocomposites

\begin{tabular}{|c|c|c|c|c|c|c|c|}
\hline \multirow{2}{*}{$\frac{\text { Sample code }}{\text { Pure cotton }}$} & \multicolumn{2}{|c|}{ Sheet resistance $\left(R_{\mathrm{s}}\right)$} & \multicolumn{2}{|c|}{ Tensile strength (mPa) } & \multirow{2}{*}{$\begin{array}{l}\text { Water contact } \\
\text { angle (WCA) }\end{array}$} & \multirow{2}{*}{$\begin{array}{l}\text { Air perme } \\
\text { ability }\left(\mathrm{L} \mathrm{m} \mathrm{m}^{-2} \mathrm{~s}^{-1}\right)\end{array}$} & \multirow{2}{*}{$\begin{array}{l}\text { Fabric } \\
\text { thickness }(\mu \mathrm{m})\end{array}$} \\
\hline & Before washing & After washing & Warp & Weft & & & \\
\hline GO cotton & 0 & 0 & 28 & 20 & 73.4 & 165 & 226.8 \\
\hline rGO cotton & 185 & 195 & 32 & 30 & 109.2 & 156 & 228.6 \\
\hline rGO-PES-1 & 165 & 175 & 39 & 35 & 121.3 & 148 & 230.4 \\
\hline rGO-PES-2 & 145 & 155 & 47 & 45 & 132.5 & 141 & 234.5 \\
\hline rGO-PES-3 & 125 & 135 & 63 & 60 & 139.4 & 165 & 236.8 \\
\hline rGO-PES-4 & 115 & 125 & 65 & 62 & 141.6 & 156 & 239.6 \\
\hline
\end{tabular}

higher water contact angles of $141.6^{\circ}$ and $139.4^{\circ}$ for distilled water droplets. The water contact angle was found to be $121.3^{\circ}$, $109.6^{\circ}$, and $73.5^{\circ}$, for different weight percent of rGO in conductive polymer nanocomposites as presented in ESI-S1 Table S2 and the ESI-S1 (ESI $\dagger$ ) measurements and characterization section Fig. S4, as compared to GO and uncoated samples, wherein the water droplet disappeared and seeped into the fabric in 1-2 seconds.

4.2.4 Tensile strength. The mechanical performances of the rGO and PEDOT:PSS-rGO samples were analysed according to ASTM standard D-5035-11 ${ }^{64}$ after the number of dyeing and washing cycles. The results indicate that the tensile strength of the fabric increased as the coating layers were increased. These changes were due to the increasing number of rGO layers on the fiber surface, resulting in an enhancement in the tensile properties of the fabric as compared to untreated samples. The tensile strength of the as-coated textile substrate was analysed in both the warp and weft directions, and the results are presented in Fig. 3(a) and (b). The results show that the mechanical performance was enhanced in both the warp and weft directions after coating with rGO layers and increasing the number of padding passes. The results also indicate that the tensile strength was slightly higher warp-wise as compared to weft-wise, which may be attributed to more ends as compared to picks in the fabric structure, which may lead to a greater loading percent of rGO on the fiber surface warp wise. ${ }^{65}$ The average tensile strength of the untreated cotton fabric was improved from $20,30,40$, and $60 \mathrm{mPa}$ for $2.5,5.0,10.0$ and 20.0 percent of rGO in the conductive polymer PEDOT: PSS by filler content in the composition. The results are presented in the ESI-S1 (ESI $\dagger$ ), see Table 2. The tensile strength improved as the concentration of rGO increased with the number of padding cycles (10). The FESEM results demonstrate that the conductive polymer PEDOT:PSS nanofilms completely covered the entire fiber surface and greater adhesion of the rGO sheets between the fiber-to-fiber conductive paths was observed from FESEM Fig. 2(a)-(c), and may be attributed to the reinforcement and inter bonding $\pi-\pi$ bonding of the PEDOT PSS-rGO nanocomposites, which improved the tensile strength of the resultant fabric surface. ${ }^{66}$

4.2.5 Fabric thickness and weight pick-up percentage. The fabric thickness was measured using an analogue thickness meter; according to ASTM standards D-1777-96 ${ }^{67}$ the samples were analysed before and after the coating with reduced graphene oxide and PEDOT:PSS. The results show that the thickness is directly proportional to the number of dyeing cycles. The thickness and weight percent of the fabric further increased when PEDOT:PSS was coated on the rGO-coated textile substrate. The weight pick-up percentage, particle size and thickness of the fabric varied from 226.8-239.6 $\mu \mathrm{m}$ for the number of rGO layers, which is attributed to increasing the content of rGO and PEDOT:PSS. The results demonstrate that the weight percent pickup, particle size and thickness of the rGO coated fabric increased with increasing the number of dyeing cycles, which resulted in the increased number of graphene and polymer layers; see ESI $\uparrow$ (ESI-S3 Fig. S2). ${ }^{68}$ In the case of the untreated fabric, the average thickness of the untreated cotton fabric was nearly $226.5 \mu \mathrm{m}$, which gradually increased from $228.6-230.4 \mu \mathrm{m}$ for the pristine $\mathrm{GO}$ and rGO-coated fabric. The thickness of the fabric was improved for PEDOT:PSS-rGO nanocomposites to $230.4 \mu \mathrm{m}, 234.5 \mu \mathrm{m}$, $236.8 \mu \mathrm{m}$, and $239.6 \mu \mathrm{m}$ for different contents of rGO as a filler in the conductive polymer with variable ratios of 2.5, 5.0, 10.0 and $20.0 \%$, respectively as shown in Table 2 .

4.2.6 Bending and stretching cycles. The developed textile electrodes coated with rGO and PEDOT:PSS with rGO nanocomposites showed a negligible difference in sheet resistance with the number of stretching and bending cycles as demonstrated in the ESI $\dagger$ (ESI-S2 Fig. S1c). The results show that the long-lasting stretching-releasing and folding-unfolding cycles of graphene and rGO-PEDOT:PSS-based nanocomposite coated TE device stably worked as an electric heater without influencing the electrical properties, and remained stable under variable temperature ranges. ${ }^{69} \mathrm{~A}$ very slight and negligible change in electric sheet resistance was attained by the fabric electrodes after applying consistent weight with 100 stretching and 500 bending cycles during folding-unfolding. The study revealed that the resultant electrodes are highly robust and stable against mechanical dynamics and are suitable as wearable heaters and thermoelectric devices. ${ }^{54}$ However, the resultant textile electrodes also showed stable behavior upon applying several folding angles $\left(0\right.$ to $\left.180^{\circ}\right)$ for each sample. ${ }^{61}$ The relatively low difference in sheet resistance was observed with a slight increase of 6-9\%, as illustrated in Fig. 1(c) and (d). The overall thermoelectric effectiveness under dynamic loading showed an improved TE performance, which significantly improved the performance of the device in terms of currentvoltage $(I-V)$ under a variable temperature range, as presented in Fig. $3(\mathrm{c})$ and (d), without any permanent deformation as reported 
in earlier studies. ${ }^{70,71}$ A nearly linear $I-V$ curve signifies the decrease in the electrical sheet resistance, which demonstrates that the electronic and electrical transport may be altered with the expansion of continuous conductive pathways of wearable thermoelectric devices. In brief, such an increase in electrical performance may be attributed to the close and compact coordinating impact of the fiber and yarn assembly decorated with graphene and a conductive polymer. ${ }^{72}$ Mechanical stretching also resulted in the dual-mode, wearable thermoelectric device, coated with rGO and PEDOT:PSS, in which PSS worked as the insulating part and binder, with the PEDOT counterpart as an improved "electrical adhesive" coated onto the rGO-treated fabric-based wearable e-textiles. ${ }^{72}$

\section{Conclusion}

The study shows that the graphene and PEDOT:PSS-coated textile fabric can potentially be used for energy harvesting from human body heat. From the study, it is evident that the production-scale dyeing of cotton fabric with reduced graphene oxide and PEDOT:PSS showed improved electrical performance due to the reduced sheet resistance from $185-25 \mathrm{k} \Omega$, and reduced thermal conductivity of $\left(0.75-0.25 \mathrm{~mW} \mathrm{~m}^{-1} \mathrm{~K}^{-1}\right)$. The resultant textilebased TE device is capable of directly converting body heat into electrical energy, with an improved Seebeck coefficient of 0.5$9.0 \times 10^{-4}$, power factor of $0.5-25.5 \mathrm{~mW} \mathrm{~m}^{-1} \mathrm{~K}^{-2}$, and thermoelectric figure of merit $(z T)$ value from $0.02-0.04 \times 10^{-4}$ as compared to the pristine rGO and PEDOT:PSS coated textile fabric. The resultant textile fabric showed enhanced thermoelectric efficiency with an output voltage of 2.5-9.5 $\mathrm{mV}$ in parallel and 12.5-19.5 $\mathrm{mV}$ in series arrangement with 2-4 TE legs connected to each other and placed over the human wrist without any masking tape. The results also demonstrate that the open circuit output voltage increased to $19.5-120.0 \mathrm{mV}$ when the number of TE legs increased to 8-10 in a series arrangement with a temperature gradient $(\Delta T)$ of $16 .{ }^{\circ} \mathrm{C} \pm 2.0{ }^{\circ} \mathrm{C}$. The study also demonstrates that the resultant coated textile fabric has a higher water contact angle in the range $93.5^{\circ}-141^{\circ}$, and mechanical performance with a tensile strength of 20-80 $\mathrm{mPa}$. The rGO-coated textile substrate showed better performance in terms of breathability and was stable against 50 washing cycles under wet and dry conditions. These attributes may be the result of the graphene-based, highly flexible, washable, breathable, and bendable textile fabric, which are only possible through the fabrication of conductive textiles using water-based GO as a dye solution. The same research approach may also potentially be used for the design and development of textile fabrics coated with rGO, PEDOT: PSS and their nanocomposites, as well as other conductive polymers for different textile substrates for future use in highly conductive and flexible sensors, actuators, energy harvesting, and storage devices.

\section{Author contributions}

N. A. Conceptualization, concept, investigation, methodology, writing and drafting original draft. W.J. J. Formal analysis, reviewing, funding, Supervision, Validation and Visualisation. X. G. Y. Resourcing and software, X. J. Resourcing and software, S. T. Resourcing and software.

\section{Conflicts of interest}

All the Authors declare no conflicts of financial and any other means.

\section{Acknowledgements}

The authors would like to acknowledge that the work is supported by China Scholarship Council.

\section{Notes and references}

1 A. Nozariasbmarz, J. H. Dycus, M. J. Cabral, C. M. Flack, J. S. Krasinski, J. M. LeBeau and D. Vashaee, Efficient selfpowered wearable electronic systems enabled by microwave processed thermoelectric materials, Appl. Energy, 2021, 283, 116211.

2 L. Jin, T. Sun, W. Zhao, L. Wang and W. Jiang, Durable and washable carbon nanotube-based fibers toward wearable thermoelectric generators application, J. Power Sources, 2021, 496, 229838.

3 L. Deng and G. Chen, Recent Progress in Tuning Polymer Oriented Microstructures for Enhanced Thermoelectric Performance, Nano Energy, 2020, 105448.

4 Z. Fan, Y. Zhang, L. Pan, J. Ouyang and Q. Zhang, Recent developments in flexible thermoelectrics: From materials to devices, Renewable Sustainable Energy Rev., 2020, 110448.

5 N. Wen, Z. Fan, S. Yang, Y. Zhao, T. Cong, S. Xu and L. Pan, Highly conductive, ultra-flexible and continuously processable PEDOT: PSS fibers with high thermoelectric properties for wearable energy harvesting, Nano Energy, 2020, 78, 105361.

6 N. Nandihalli, C. J. Liu and T. Mori, Polymer based thermoelectric nanocomposite materials and devices: Fabrication and characteristics, Nano Energy, 2020, 105186.

7 L. S. Zhang, B. Yang, S. P. Lin, T. Hua and X. M. Tao, Predicting performance of fiber thermoelectric generator arrays in wearable electronic applications, Nano Energy, 2020, 76, 105117.

8 L. Jin, T. Sun, W. Zhao, L. Wang and W. Jiang, Durable and washable carbon nanotube-based fibers toward wearable thermoelectric generators application, J. Power Sources, 2021, 496, 229838.

9 A. Lund, Y. Tian, S. Darabi and C. Müller, A polymer-based textile thermoelectric generator for wearable energy harvesting, J. Power Sources, 2020, 480, 228836.

$10 \mathrm{~J}$. Yuan and R. Zhu, A fully self-powered wearable monitoring system with systematically optimized flexible thermoelectric generator, Appl. Energy, 2020, 271, 115250.

11 J. Choi, Y. Jung, C. Dun, K. T. Park, M. P. Gordon, K. Haas and J. J. Urban, High-performance, wearable thermoelectric 
generator based on a highly aligned carbon nanotube sheet, ACS Appl. Energy Mater., 2019, 3(1), 1199-1206.

12 M. Bharti, A. Singh, B. P. Singh, S. R. Dhakate, G. Saini, S. Bhattacharya and D. K. Aswal, Free-standing flexible multiwalled carbon nanotubes paper for wearable thermoelectric power generator, J. Power Sources, 2020, 449, 227493.

13 X. Jia and Q. Guo, Design study of Bismuth-Telluride-based thermoelectric generators based on thermoelectric and mechanical performance, Energy, 2020, 190, 116226.

14 H. Hardianto, G. D. Mey, B. Malengier and L. V. Langenhove, Textile-based thermoelectric generator fabricated from carbon fibers, J. Ind. Text., 2020, 1528083720910686.

15 I. Petsagkourakis, K. Tybrandt, X. Crispin, I. Ohkubo, N. Satoh and T. Mori, Thermoelectric materials and applications for energy harvesting power generation, Sci. Technol. Adv. Mater., 2018, 19(1), 836-862.

16 J. Ren, C. Wang, X. Zhang, T. Carey, K. Chen, Y. Yin and F. Torrisi, Environmentally-friendly conductive cotton fabric as flexible strain sensor based on hot press reduced graphene oxide, Carbon, 2017, 111, 622-630.

17 H. Kim and J. H. Ahn, Graphene for flexible and wearable device applications, Carbon, 2017, 120, 244-257.

18 L. Hu, M. Pasta, F. La Mantia, L. Cui, S. Jeong, H. D. Deshazer and Y. Cui, Stretchable, porous, and conductive energy textiles, Nano Lett., 2010, 10(2), 708-714.

19 S. H. Aboutalebi, R. Jalili, D. Esrafilzadeh, M. Salari, Z. Gholamvand, S. Aminorroaya Yamini and P. C. Innis, Highperformance multifunctional graphene yarns: toward wearable allcarbon energy storage textiles, ACS Nano, 2014, 8(3), 2456-2466.

20 G. Acar, O. Ozturk, A. J. Golparvar, T. A. Elboshra, K. Böhringer and M. K. Yapici, Wearable and flexible textile electrodes for biopotential signal monitoring: A review, Electronics, 2019, 8(5), 479.

21 V. Kaushik, J. Lee, J. Hong, S. Lee, S. Lee, J. Seo and T. Lee, Textile-based electronic components for energy applications: principles, problems, and perspective, Nanomaterials, 2015, 5(3), 1499-1531.

22 W. Weng, P. Chen, S. He, X. Sun and H. Peng, Smart electronic textiles, Angew. Chem., Int. Ed., 2016, 55(21), 6140-6169.

23 B. Wang and A. Facchetti, Mechanically Flexible Conductors for Stretchable and Wearable E-Skin and E-Textile Devices, Adv. Mater., 2019, 31(28), 1901408.

24 K. S. Novoselov, V. I. Fal, L. Colombo, P. R. Gellert, M. G. Schwab and K. Kim, A roadmap for graphene, Nature, 2012, 490(7419), 192-200.

25 D. Lee, H. Lee, Y. Ahn, Y. Jeong, D. Y. Lee and Y. Lee, Highly stable and flexible silver nanowire-graphene hybrid transparent conducting electrodes for emerging optoelectronic devices, Nanoscale, 2013, 5(17), 7750-7755.

26 M. Mirjalili, Preparation of electroconductive, magnetic, antibacterial, and ultraviolet-blocking cotton fabric using reduced graphene oxide nanosheets and magnetite nanoparticles, Fibers Polym., 2016, 17(10), 1579-1588.

27 Y. Zhang, Q. Xu, F. Fu and X. Liu, Durable antimicrobial cotton textiles modified with inorganic nanoparticles, Cellulose, 2016, 23(5), 2791-2808.
28 N. D. Tissera, R. N. Wijesena, J. R. Perera, K. N. de Silva and G. A. Amaratunge, Hydrophobic cotton textile surfaces using an amphiphilic graphene oxide (GO) coating, Appl. Surf. Sci., 2015, 324, 455-463.

29 Y. Ji, Y. Li, G. Chen and T. Xing, Fire-resistant and highly electrically conductive silk fabrics fabricated with reduced graphene oxide via dry-coating, Mater. Des., 2017, 133, 528-535.

30 L. Karimi, S. Zohoori and A. Amini, Multi-wall carbon nanotubes and nano titanium dioxide coated on cotton fabric for superior self-cleaning and UV blocking, New Carbon Mater., 2014, 29(5), 380-385.

31 J. N. Chakraborty, M. R. Mohapatra and J. Kumar, Differential functional finishes for textiles using graphene oxide, Res. J. Text. Apparel, 2018, 22(1), 77-91.

32 M. Montazer and S. Seifollahzadeh, Enhanced self-cleaning, antibacterial and UV protection properties of nano $\mathrm{TiO} 2$ treated textile through enzymatic pretreatment, Photochem. Photobiol., 2011, 87(4), 877-883.

33 D. Madan, A. Chen, P. K. Wright and J. W. Evans, Printed Sedoped MA n-type Bi 2 Te 3 thick-film thermoelectric generators, J. Electron. Mater., 2012, 41(6), 1481-1486.

34 D. Madan, Z. Wang, P. K. Wright and J. W. Evans, Printed flexible thermoelectric generators for use on low levels of waste heat, Appl. Energy, 2015, 156, 587-592.

35 N. Y. Abu-Thabit and A. S. H. Makhlouf, Smart Textile Supercapacitors Coated with Conducting Polymers for Energy Storage Applications. Industrial Applications for Intelligent Polymers and Coatings, Springer, Cham, 2016, pp. 437-477.

36 L. C. Jia, K. Q. Ding, R. J. Ma, H. L. Wang, W. J. Sun, D. X. Yan and Z. M. Li, Highly Conductive and MachineWashable Textiles for Efficient Electromagnetic Interference Shieldinr, Adv. Mater. Technol., 2019, 4(2), 1800503.

37 L. Liu, Y. Yu, C. Yan, K. Li and Z. Zheng, Wearable energydense and power-dense supercapacitor yarns enabled by scalable graphene-metallic textile composite electrodes, Nat. Commun., 2015, 6(1), 1-9.

38 L. Karimi, S. Zohoori and A. Amini, Multi-wall carbon nanotubes and nano titanium dioxide coated on cotton fabric for superior self-cleaning and UV blocking, New Carbon Mater., 2014, 29(5), 380-385.

39 T. Carey, S. Cacovich, G. Divitini, J. Ren, A. Mansouri, J. M. Kim and F. Torrisi, Fully inkjet-printed twodimensional material field-effect heterojunctions for wearable and textile electronics, Nat. Commun., 2017, 8(1), 1-11.

40 Y. Shao, M. F. El-Kady, L. J. Wang, Q. Zhang, Y. Li, H. Wang and R. B. Kaner, Graphene-based materials for flexible supercapacitors, Chem. Soc. Rev., 2015, 44(11), 3639-3665.

41 D. S. Montgomery and D. L. Carroll, Improving upon flexible thin film thermoelectric generator design, coupled thermoelectric and piezoelectric meta structures for renewable energy generation, 2016, vol. 96, p. 56.

42 C. Navone, M. Soulier, M. Plissonnier and A. L. Seiler, Development of (Bi, Sb) 2 (Te, Se) 3-based thermoelectric modules by a screen-printing process, J. Electron. Mater., 2010, 39(9), 1755-1759.

43 M. A. Shathi, C. Minzhi, N. A. Khoso, H. Deb, A. Ahmed and W. S. Sai, All organic graphene oxide and Poly (3, 4-ethylene 
dioxythiophene)-Poly (styrene sulfonate) coated knitted textile fabrics for wearable electrocardiography (ECG) monitoring, Synth. Met., 2020, 263, 116329.

44 T. Sun, J. J. Wang, N. A. Khoso, L. Yu and Y. Zhang, Facile synthesis of Poly (3, 4-ethylenedioxythiophene) nanostructure with controlled morphologies by using an aqueous surfactant soft-template-assisted technique, Mater. Lett., 2017, 191, 61-64.

45 Y. C. Sun, Development and Characterization of Next Generation Flexible Dielectric and Thermoelectric Energy Harvesting Materials, Doctoral dissertation, 2015.

46 M. Thielen, L. Sigrist, M. Magno, C. Hierold and L. Benini, Human body heat for powering wearable devices: From thermal energy to application, Energy Convers. Manage., 2017, 131, 44-54.

47 M. Tian, X. Hu, L. Qu, M. Du, S. Zhu, Y. Sun and G. Han, Ultraviolet protection cotton fabric achieved via layer-bylayer self-assembly of graphene oxide and chitosan, Appl. Surf. Sci., 2016, 377, 141-148.

48 M. Tian, X. Hu, L. Qu, S. Zhu, Y. Sun and G. Han, Versatile and ductile cotton fabric achieved via layer-by-layer selfassembly by consecutive adsorption of graphene doped PEDOT: PSS and chitosan, Carbon, 2016, 96, 1166-1174.

49 J. Vondrak, M. Schmidt, A. Proto, M. Penhaker, J. Jargus and L. Peter, Using Miniature Thermoelectric Generators for Wearable Energy Harvesting, 2019 4th International Conference on Smart and Sustainable Technologies (SpliTech), IEEE, 2019, pp. 1-6.

50 W. Zeng, L. Shu, Q. Li, S. Chen, F. Wang and X. M. Tao, Fiber-based wearable electronics: a review of materials, fabrication, devices, and applications, Adv. Mater., 2014, 26(31), 5310-5336.

51 H. P. Wong and Z. Dahari, Human body parts heat energy harvesting using thermoelectric module, 2015 IEEE Conference on Energy Conversion (CENCON), IEEE, 2015, pp. 211-214.

52 J. Hamada, K. Yamamoto and M. Takashiri, Fabrication and characterization of roll-type thin-film thermoelectric generators, Journal of Physics: Conference Series, IOP Publishing, 2018, vol. 1052, No. 1, p. 012129.

53 Z. Zhu, C. Liu, Q. Jiang, H. Shi, J. Xu, F. Jiang and E. Liu, Green DES mixture as a surface treatment recipe for improving the thermoelectric properties of PEDOT: PSS films, Synth. Met., 2015, 209, 313-318.

54 L. Dong, C. Xu, Y. Li, C. Wu, B. Jiang, Q. Yang and Q. H. Yang, Simultaneous Production of High-Performance Flexible Textile Electrodes and Fiber Electrodes for Wearable Energy Storage, Adv. Mater., 2016, 28(8), 1675-1681.

55 Y. Du, J. Xu, Y. Wang and T. Lin, Thermoelectric properties of graphite-PEDOT: PSS coated flexible polyester fabrics, J. Mater. Sci.: Mater. Electron., 2017, 28(8), 5796-5801.

56 M. T. Dunham, M. T. Barako, J. E. Cornett, Y. Gao, S. Haidar, N. Sun and K. E. Goodson, Experimental characterization of microfabricated thermoelectric energy harvesters for smart sensor and wearable applications, Adv. Mater. Technol., 2018, 3(6), 1700383.

57 Y. Huang, H. Hu, Y. Huang, M. Zhu, W. Meng, C. Liu and C. Zhi, From industrially Weavable and knittable highly conductive yarns to large wearable energy storage textiles, ACS Nano, 2015, 9(5), 4766-4775.

58 M. Hyland, H. Hunter, J. Liu, E. Veety and D. Vashaee, Wearable thermoelectric generators for human body heat harvesting, Appl. Energy, 2016, 182, 518-524.

59 M. Z. Islam, Y. Dong, N. A. Khoso, A. Ahmed, H. Deb, Y. Zhu and $\mathrm{Y}$. Fu, Continuous dyeing of graphene on cotton fabric: Binder-free approach for electromagnetic shielding, Appl. Surf. Sci., 2019, 496, 143636.

60 Y. S. Jung, D. H. Jeong, S. B. Kang, F. Kim, M. H. Jeong, K. S. Lee and K. J. Choi, Wearable solar thermoelectric generator driven by unprecedentedly high temperature difference, Nano Energy, 2017, 40, 663-672.

61 N. Karim, S. Afroj, S. Tan, P. He, A. Fernando, C. Carr and K. S. Novoselov, Scalable production of graphene-based wearable e-textiles, ACS Nano, 2017, 11(12), 12266-12275.

62 X. Pu, M. Liu, L. Li, S. Han, X. Li, C. Jiang and Z. L. Wang, Wearable textile-based in-plane microsupercapacitors, $A d v$. Energy Mater., 2016, 6(24), 1601254.

63 I. A. Sahito and A. Khatri, Smart and Electronic Textiles, Advanced Textile Testing Techniques, CRC Press, 2017, pp. 309-328.

64 I. A. Sahito, K. C. Sun, A. A. Arbab, M. B. Qadir and S. H. Jeong, Graphene coated cotton fabric as textile structured counter electrode for DSSC, Electrochim. Acta, 2015, 173, 164-171.

65 M. Shateri-Khalilabad and M. E. Yazdanshenas, Preparation of superhydrophobic electroconductive graphene-coated cotton cellulose, Cellulose, 2013, 20(2), 963-972.

66 V. Kaushik, J. Lee, J. Hong, S. Lee, S. Lee, J. Seo and T. Lee, Textile-based electronic components for energy applications: principles, problems, and perspective, Nanomaterials, 2015, 5(3), 1493-1531.

67 Z. Lin, C. Hollar, J. S. Kang, A. Yin, Y. Wang, H.-Y. Shiu, Y. Huang, Y. Hu, Y. Zhang and X. Duan., A Solution Processable High-Performance Thermoelectric Copper Selenide Thin Film, Adv. Mater., 2017, 29(no. 21), 1606662.

68 https://www.astm.org/Standards/textile-standards.html.

69 N. A. Khoso, A. Ahmed, H. Deb, S. Tian, X. Jiao, X. Y. Gong and J. Wang, Controlled template-free in-situ polymerization of PEDOT for enhanced thermoelectric performance on textile substrate, Org. Electron., 2019, 75, 105368.

70 M. K. Kim, M. S. Kim, S. Lee, C. Kim and Y. J. Kim, Wearable thermoelectric generator for harvesting human body heat energy, Smart Mater. Struct., 2014, 23(10), 105002.

71 S. H. Lee, H. Park, W. Son, H. H. Choi and J. H. Kim, Novel solution-processable, dedoped semiconductors for application in thermoelectric devices, J. Mater. Chem. A, 2014, 2(33), 13380-13387.

72 H. Liu, Y. Wang, D. Mei, Y. Shi and Z. Chen, Design of a wearable thermoelectric generator for harvesting human body energy, Wearable sensors and robots, Springer, Singapore, 2017, pp. 55-66.

73 H. Yu, B. Zhang, C. Bulin, R. Li and R. Xing, High-efficient synthesis of graphene oxide based on improved hummers method, Sci. Rep., 2016, 6(1), 36143. 